\title{
La « révolution » thatchérienne en perspective : l'Angleterre et ses pauvres depuis 1834
}

\author{
Jacques Rodriguez
}

\section{(2) OpenEdition \\ 1 Journals}

\section{Édition électronique}

URL : http://journals.openedition.org/conflits/177

DOI : 10.4000/conflits. 177

ISSN : $1777-5345$

Éditeur :

CCLS - Centre d'études sur les conflits lilberté et sécurité, L'Harmattan

\section{Édition imprimée}

Date de publication : 1 septembre 1999

ISBN : 2-7384-8616-9

ISSN : 1157-996X

\section{Référence électronique}

Jacques Rodriguez, « La « révolution» thatchérienne en perspective : I'Angleterre et ses pauvres depuis 1834 », Cultures \& Conflits [En ligne], 35 | automne 1999, mis en ligne le , consulté le 30 mars 2021. URL : http://journals.openedition.org/conflits/177 ; DOI : https://doi.org/10.4000/conflits. 177

Ce document a été généré automatiquement le 30 mars 2021.

Creative Commons License 


\title{
La « révolution » thatchérienne en perspective : l'Angleterre et ses pauvres depuis 1834
}

\author{
Jacques Rodriguez
}

En matière de politique sociale, le thatchérisme est désormais une référence incontournable. Le dénigrement de la politique sociale des Conservateurs britanniques est devenu un lieu commun, une clause de style dans de nombreux débats, un anathème que l'on jette sur ceux qui professent le credo libéral - et qu'ils repoussent d'ailleurs, tant la filiation leur paraît lourde à assumer. Pourtant le sens et la portée de la "révolution thatchérienne » sont rarement précisés; ils sont au contraire obscurcis par l'exploitation souvent polémique de toute référence à cette période de l'histoire anglaise. La politique sociale conduite par les gouvernements conservateurs à partir de 1979, avec laquelle la nouvelle majorité travailliste ne semble pas avoir rompu, s'est effectivement traduite par une progression de la pauvreté. Sur le long terme, les chiffres sont dépourvus d'ambiguïté : tandis que $9 \%$ de la population vivait avec des ressources inférieures à la moitié du revenu moyen en 1979, 25\% des britanniques seront dans cette situation en 1992, soit environ 14 millions de personnes. Après que le second gouvernement travailliste d'Harold Wilson (1974-76) ait amorcé une politique de baisse des dépenses sociales, les gouvernements conservateurs vont poursuivre et accentuer cette tendance en mobilisant tous les topiques de la «rhétorique réactionnaire " patiemment élaborée dans l'enceinte des think tanks néo-libéraux. L'intervention sociale de l'Etat sera dès lors présentée comme une source d' "effets pervers ": son coût élevé pénaliserait l'investissement productif et compromettrait la reprise économique; le niveau des prestations diminuerait l'incitation au travail et placerait nombre d'individus dans le "piège de la pauvreté». Par un singulier paradoxe, l'action de l'Etat menacerait ainsi d'appauvrir les plus pauvres; elle contribuerait à disloquer les familles et, surtout, elle produirait des "parasites sociaux» (scroungers), habiles profiteurs d'un système inutilement lourd et exagérément généreux. Amplifiant les effets dévastateurs de la récession économique du début des années 1980 , la réduction des dépenses sociales a notablement précarisé la 
situation des personnes les plus fragiles ${ }^{1}$. Toutefois, les réformes entreprises par les Conservateurs ne se sont pas traduites par un démantèlement du Welfare State d'aprèsguerre - même si cela a pu figurer parmi leurs objectifs. On observe en particulier que les dépenses consacrées au système de santé public (le National Health Service) - dont le caractère universel et égalitaire est, selon T.H. Marshall, l'incarnation même de la « logique » de l'Etat-providence ${ }^{2}$ - ont progressé d'un tiers au cours des années 1980, et qu'elles représentaient plus de $16 \%$ des dépenses publiques en 1990 , contre $14 \%$ en 1979. En revanche ces réformes se sont efforcées de réajuster les interventions sociales de manière à placer chacun en position de "gagner » son autonomie. "Laissez moi vous donner ma vision des choses ", dira Margaret Thatcher aux responsables du parti conservateur réunis à Blackpool en 1975: "un homme a le droit de travailler, de dépenser ce qu'il gagne, de devenir propriétaire, il doit être servi par l'Etat et ne pas lui être asservi ; tel est l'héritage britannique ». Son propos vise implicitement les assistés du Welfare State, en particulier les chômeurs, accusés de s'être installés dans l'oisiveté. En effet, cet « héritage britannique » qu'elle évoque recouvre moins les droits reconnus à ceux qui travaillent que les contraintes imposées aux personnes dépourvues de ressources. En stigmatisant telle ou telle catégorie de bénéficiaires de la protection sociale, elle ne fait que réactiver le schéma du « mauvais pauvre ", dont la situation ne résulterait pas de causes objectives mais d'une moralité douteuse, d'un comportement atavique ou d'une malice coupable. Il s'agit là d'un thème puissant et récurrent dans l'histoire sociale anglaise. C'est pourquoi on s'efforcera de montrer qu'en matière de traitement de la pauvreté la «révolution thatchérienne " ressemble fort, dans sa logique, à ce que Beveridge appelait une "révolution à l'anglaise »: elle capitalise un héritage et couronne une évolution. Cet article s'attachera donc moins à décrire les conséquences dévastatrices et bien connues de cette politique qu'à explorer ses sources et à l'inscrire dans un mouvement de fond depuis l'Angleterre victorienne. En retraçant l'itinéraire de la catégorie du "mauvais pauvre», il en rappellera les conditions d'émergence et soulignera le rôle ambigu de la réflexion (pré)sociologique dans la construction de la citoyenneté sociale et dans la substitution du critère du besoin à celui du mérite comme régulateur des interventions sociales en faveur des pauvres. La pesanteur du stigmate attaché à la condition d'assisté, sans cesse réduite à une citoyenneté sociale au rabais sur laquelle plane le soupçon de la fraude ou de l'imposture, permettra d'isoler un trait culturel particulièrement robuste dans une société acquise à l'éthique du travail. A chacun selon son mérite Le pauvre "non méritant" entre en scène au XIXème siècle. A cette époque, les responsables gouvernementaux sont inquiets des transformations endogènes de la loi des pauvres élisabéthaine (1601). Premier dispositif d'envergure nationale, cette loi assurait, dans des conditions globalement satisfaisantes, la prise en charge des pauvres dans leur paroisse de résidence. Toutefois la croissance des dépenses consacrées à l'assistance, en particulier lors des guerres napoléoniennes, suscite une vive émotion parmi les défenseurs de la nouvelle économie libérale. Mais ce sont surtout les effets prétendument pernicieux d'une assistance systématique (le système de Speenhamland, 1795), qui retiendront l'attention des réformateurs. « La somme des bienfaits à attendre de l'établissement d'une assistance obligatoire aux pauvres sera plus que dépassée par l'ensemble des méfaits que cela produira. La certitude d'un secours prévu par la loi affaiblira en effet les principes de l'affection naturelle et détruira le plus puissant des liens sociaux en rendant moins nécessaire l'exercice des devoirs domestiques et sociaux " ${ }^{3}$. Même sous la plume d'un philanthrope modéré comme Morton Eden, un 
système d'aide indiscriminée est accusé de déstabiliser la cellule familiale et les réseaux de solidarité charpentés par l'affection ou la sociabilité de proximité. Maintes fois formulées, de telles critiques provoquèrent la réunion d'une commission d'experts en charge d'une énième réforme de la loi de 1601. Nassau Senior, Edwin Chadwick et les membres de la Commission royale de 1832-34 estimeront que les paroisses ne doivent en aucun cas «jouer le rôle d'assureur universel de toutes les classes de la société contre les méfaits de l'imprévoyance et de la paresse " ${ }^{4}$, sous peine de "démoraliser " les pauvres et de déprimer la productivité du travail. Ils pensaient en effet que la certitude d'obtenir un secours, sous forme de complément de salaire, incitait les travailleurs à la flânerie. Le système ne devait en aucun cas garantir à chacun un «droit» à l'assistance: il devait au contraire être révisé de manière à inciter fermement les pauvres à devenir indépendants, responsables et économes. Et l'on observe d'ailleurs que les victoriens défendront avec constance les vertus de l'épargne et la constitution de sociétés d'entraide, les friendly societies particulièrement nombreuses dans l'Angleterre du XIXème siècle. On devine la théorie implicite de l'intégration qui anime les réflexions de ces réformateurs: c'est par le travail et l'insertion dans le réseau des échanges économiques que chacun trouvera sa place dans la société, pourra conquérir son indépendance et se dégager des griffes du «servage paroissial ». Le travail n'est-il pas, en effet, « le père de la richesse », « le plus grand des biens " ${ }^{5}$, grâce auquel le processus de paupérisation peut être enrayé ? Il permet le développement des échanges, la multiplication et la diffusion des richesses; il autorise la constitution d'une épargne, favorise la prévoyance et l'autonomie individuelle. Compte tenu des opportunités que le travail est censé offrir au travailleur industrieux et prévoyant, la pauvreté ne peut résulter que de défaillances personnelles ou d'un choix rationnel. Selon les réformateurs, seuls deux cas de figures peuvent se présenter. S'il est physiquement inapte au travail, le vieillard, l'infirme ou l'enfant privé d'attaches familiales doit être (chichement) secouru par la collectivité. Mais en revanche le pauvre valide, dont on pense qu'il refuse le travail ou en dilapide inconsidérément les fruits, est un individu nécessairement immoral, paresseux ou simulateur, qui ne "mérite» donc aucune compassion et doit être traité avec la plus extrême fermeté. La réflexion des réformateurs britanniques de 1834 est ainsi soustendue par le paradigme de l'indigence "volontaire " ${ }^{6}$, occultant complètement le contexte économique et social pour traquer les comportements déviants. L'indigent valide, accusé de se dérober volontairement aux contraintes du travail pour tirer parti d'une assistance trop peu sélective, incarne ce «mauvais pauvre » dont la nouvelle loi des pauvres doit désormais normaliser le comportement. C'est à partir de telles prémisses que la New Poor Law de 1834 va réviser en profondeur la vieille loi élisabéthaine. Elle concevra l'assistance comme une peine, qui ne traduit pas un droit, mais exige des devoirs : un devoir-faire et un devoir-être. C'est pourquoi toute aide (à l'exception des secours médicaux d'urgence) devra être octroyée dans l'enceinte d'une workhouse, dont les pensionnaires seront immédiatement mis au travail. Afin de les dissuader de solliciter l'assistance publique et, corrélativement, les inciter à rester sur le marché du travail, les conditions de vie offertes par la workhouse doivent être "moins attractives» (principe de less eligibility) que celles dont peut disposer le plus humble des travailleurs indépendants. De cette façon les contraintes imposées à l'assisté sont censées tester de manière infaillible la réalité de ses besoins : l'indigent ne se soumettra à la discipline de la workhouse que s'il est réduit à la dernière extrémité. La workhouse sera alors un espace institutionnel consacré au dressage et à la 
rééducation de tous ceux qui n'ont pas pu s'imposer l'autodiscipline que doit impliquer la simple menace de la workhouse; pour ceux-là, la «maison de travail» doit être «le surveillant le plus sévère et le pire des payeurs " ${ }^{7}$. A la monotonie et la pénibilité du travail s'ajouteront la séparation des familles, l'absence de toute vie privée, la perte des droits politiques, et la stigmatisation (le vêtement des résidents des workhouses portait souvent la lettre ' $\mathrm{P}$ ' cousue dans un tissu de couleur lumineuse). Autour de la figure de l'indigent valide, la loi de 1834 construit ainsi une catégorie de citoyens de deuxième classe, privés de liberté, de dignité ; privés aussi de leurs droits civiques et politiques. Naturellement la mise en œuvre d'un tel dispositif s'est avérée problématique: le nombre de workhouses n'était pas suffisant pour accueillir les indigents; les instructions envoyées par Londres n'étaient pas toujours suivies au niveau local; surtout, il était bien souvent difficile d'aménager le régime disciplinaire en fonction du "mérite» de chacun, et la plus grande confusion régnait dans ces établissements. Néanmoins, l'hégémonie de ce schéma interprétatif ne sera jamais contestée tout au long du XIXème siècle. On peut observer par exemple que toutes les initiatives déployées par les sociétés charitables, jusqu'en 1914 au moins, s'organiseront autour de la polarité méritant/non méritant. Les multiples associations philanthropiques s'acharneront en effet à isoler les pauvres dignes d'être secourus. La technique inquisitoriale, à laquelle elles subordonneront toute assistance, permettra d'apprécier la moralité du demandeur et d'évaluer sa responsabilité dans la situation qui l'affecte. " Notre intention est de supprimer la charité à destination des inutiles et de la canaliser vers les pauvres méritants » expliquera l'un des responsables de la Charity Organisation Society ${ }^{8}$. La philanthropie "scientifique" que veut incarner la C.O.S. se donne pour objectif de départager le "bon pauvre", victime des circonstances, de la masse des fainéants dont la démarche traînante, l'aspect négligé ou l'attitude arrogante signalera à l'observateur avisé et expérimenté l'incurable déchéance. Ceux-là seront laissés aux «bons soins » de la loi des pauvres, tandis que les autres, jugés à même de recouvrer leur indépendance, seront placés sous la tutelle bienveillante de travailleurs sociaux bénévoles. La même attitude se retrouvera à la fin du XIXème siècle lorsque les gestionnaires de l'assistance publique assoupliront les conditions d'assistance offertes aux travailleurs désœuvrés : les tenants de la charité scientifique leur opposeront que de telles initiatives risquent de "démoraliser " les bénéficiaires, c'est-à-dire d'exercer un effet désincitatif au travail. La philosophie de 1834 reposait donc sur deux principes simples. Premièrement, la pauvreté était une difficulté transitoire. En effet, le traitement de l'indigence d'un côté, et les avantages d'une insertion réussie dans la division du travail de l'autre côté, devaient automatiquement en réduire l'étendue. Deuxièmement, l'indigence était l'expression d'un choix rationnel. Demander assistance à la paroisse ne signifiait pas que le travailleur avait été évincé du marché du travail, mais qu'il avait décidé d'en refuser la rigueur (le travail) ou la sanction (le salaire). Ces deux idées étaient d'ailleurs parfaitement articulées l'une à l'autre: la pauvreté étant pensée comme un phénomène macrosocial évanescent, son expression individuelle reflétait nécessairement le comportement fautif du pauvre. L'absence de mérite désigne donc une pathologie : le pauvre valide est dépourvu de moralité et de dignité ; c'est un usurpateur qui fraude le « vrai » nécessiteux. Il figure l'image inversée de l'éthique du travail et du Self Help à laquelle le XIXème siècle anglais adhère sans réserve. Un tel schéma de perception aura encore de nombreux adeptes en Angleterre tout au long du XXème siècle. En effet, au-delà de sa dimension punitive et du traitement carcéral auquel elle soumet l'indigent déchu, la loi des pauvres a défini une 
«mentalité »; plus exactement, elle a subordonné les interventions sociales à un schéma normatif rigide où la déliquescence du rapport au travail doit être perçue comme suspecte. Cette interprétation de la réalité sociale exercera une influence durable sur la politique sociale, et des penseurs aussi résolument progressistes que Beatrice et Sidney Webb, ardents partisans de l'abandon de la loi des pauvres, resteront prisonniers d'une approche moralisante: le comportement, le caractère, l'hexis corporelle, et le rapport plus ou moins distancié au travail permettent d'isoler les fainéants « incorrigibles » des pauvres de droit commun pour placer les premiers dans des "colonies de détention" ${ }^{9}$. Ce schéma analytique deviendra une caractéristique forte de la politique sociale anglaise, qui en imprégnera les orientations même après la suppression de la Poor Law. Ambitions et ambiguités des sciences sociales A l'encontre du béhaviorisme simpliste de la loi des pauvres, les enquêteurs sociaux de la fin du XIXème et du début du XXème siècle vont proposer une représentation alternative de la pauvreté. Dans l'ensemble des travaux consacrés à ce phénomène, ceux de Charles Booth, Seebhom Rowntree ou, quelques années plus tard, ceux de Florence Bell ou Arthur Bowley auront un impact déterminant sur les orientations ultérieures de la politique sociale. Jusque dans les années 1890 en effet, c'est dans le registre mineur du reportage journalistique que l'on trouve les évocations les plus puissantes de la pauvreté : les travaux d'Henry Mayhew, de George Sims ou d'Andrew Mearns ${ }^{10}$, par exemple, eurent un réel retentissement, mais leur analyse des classes populaires était par trop impressionniste. Surtout, en ne parvenant pas à donner une représentation objective du processus de paupérisation, ces travaux n'invalidaient pas le paradigme de l'indigence "volontaire». Tout autre sera l'impact des grandes enquêtes sociales publiées à la charnière de la période edwardienne et pendant l'entre-deux-guerres. Ces travaux vont révéler l'existence d'une pauvreté structurelle, plaçant quantité d'individus en situation de grande précarité. Leur impact théorique et politique sera réel, même s'ils ne sont pas parvenus à s'affranchir totalement du schéma de la faute personnelle. Dans leur effort pour cartographier la pauvreté, Charles Booth et Seebhom Rowntree parviendront à des conclusions alarmantes : $30 \%$ des habitants de la capitale britannique vivent en situation de "pauvreté », voire de "grande pauvreté » (soit avec des ressources inférieures ou égales à une vingtaine de shillings hebdomadaires pour une "famille moyenne »); dans une ville moyennement prospère comme York, Rowntree découvre que $28 \%$ des résidents ne disposent pas des ressources nécessaires au maintien de leur « efficience physique ». Ces chiffres contrastent fortement avec les statistiques de la pauvreté assistée : au cours des années 1890 en effet, moins de 3\% des Britanniques ont été secourus par le Poor Law Board. Au-delà du périmètre de l'indigence, il existe donc une pauvreté massive privant quantité d'individus de moyens d'existence décents. Qui sont ces parias des cités industrielles? Sont-ils des pauvres " non méritants » suspectés d'immoralisme ou dissuadés de recourir à la workhouse? Les résultats avancés par les enquêteurs sont à cet égard très instructifs. Contrairement à nombre de leurs prédécesseurs, ces auteurs parviennent à mesurer précisément les causes de la pauvreté et à identifier les processus qui y conduisent. A partir d'un échantillon de 4000 familles de l'East-End londonien, Booth montrera ainsi que la " grande pauvreté » (les « classes » A et B de l'auteur) est principalement causée par le sous-emploi (55\%), tandis que les comportements répréhensibles n'interviennent qu'à hauteur de $14 \%$; s'agissant des individus classés comme " pauvres » (« classes »C et D), les « questions liées à l'emploi » exercent un impact décisif (68\%). Dans plus de $20 \%$ des cas, la maladie ou l'infirmité sont responsables de la misère ${ }^{11} \cdot{ }^{12}$. De son côté, Rowntree 
insistera sur la faiblesse des salaires et la structure des familles. Dépassant les travaux de son devancier, il parviendra à mettre en évidence l'existence d'un «cycle de pauvreté» mesurant l'intensité de celle-ci en fonction des âges de la vie et, corrélativement, de la taille des familles. C'est à l'âge adulte, avant que le premier enfant ne vienne contribuer aux ressources familiales, que le risque de paupérisation s'accroît, avant de réapparaître lorsque la vieillesse affecte la capacité de travail des individus ${ }^{13}$. Ces auteurs insistent donc sur la dimension structurelle de la pauvreté : le travail intermittent ou les bas salaires, résultant tous deux des dysfonctionnements du marché du travail, le sous-emploi chronique, les charges familiales, le grand âge, sont autant de facteurs indépendants de la personne du pauvre et dont il ne saurait être tenu responsable. Ils donnent ainsi à la pauvreté une certaine visibilité en tant que phénomène social détaché de ses expressions individuelles. Sous réserve de quelques nuances, ils présentent la pauvreté comme une forme de vulnérabilité sociale: le pauvre est soumis au poids de la nécessité, incapable d'anticiper et de se protéger contre les aléas de l'existence. Les pauvres que décrit Rowntree ne sont pas tous imprévoyants comme le supposent les administrateurs de la Poor Law; ils ne sont ni avides de sensations, ni soumis à la dictature de l'instant: ils sont structurellement condamnés à l'immédiateté. Une famille vivant conformément au barème établi (...) ne doit jamais dépenser un penny pour un billet de train ou d'omnibus. Ses membres ne doivent jamais aller à la campagne, sauf à pied. Ils ne doivent jamais acheter un journal bon marché ou dépenser un penny pour un concert populaire. Ils ne doivent jamais écrire aucune lettre à des enfants absents, car ils n'ont pas les moyens de payer l'affranchissement. (...) Si un enfant tombe malade, il doit être soigné par le médecin paroissial, et s'il meurt, il doit être enterré aux frais de la paroisse. Enfin, l'homme ne doit jamais être absent de son travail, ne serait-ce qu'un jour. (...) Une dépense supplémentaire ne sera couverte qu'en réduisant l'alimentation; ou, en d'autres termes, en sacrifiant l'efficacité physique " ${ }^{14}$. La pauvreté révélée par les enquêteurs exprime donc une vulnérabilité sociale sur laquelle se concentrent tous les risques sociaux, le chômage, la vieillesse, la maladie. Florence Bell souligne bien cette précarité de l'existence, même chez les travailleurs régulièrement employés dans la métallurgie de Middlesbrough: "S'il tombe malade, le contremaître recevra l'intégralité de son traitement pendant les deux premières semaines de son absence, mais pas l'ouvrier ordinaire. Même s'il est membre d'une association d'entraide, son revenu sera réduit au moment où il aurait dû progresser ; la nourriture et les remèdes qui lui sont nécessaires seront inaccessibles sans sacrifier quelque chose d'essentiel au bien être du reste de la famille ${ }^{15}$. Ces travaux montrent que la pauvreté est un problème global engageant la société tout entière : menaçant l'aptitude physique des individus elle affecte du même coup leur potentiel productif en tant que travailleurs. La pauvreté n'apparaît plus seulement comme l'indice d'une défaillance individuelle mais constitue un gaspillage pour la collectivité. Il est donc vain, et même contre-productif, de durcir volontairement les conditions d'assistance pour combattre la pauvreté. Au contraire, pour lutter efficacement contre celle-ci et éviter qu'elle n'altère gravement la vigueur des travailleurs ou la santé des enfants, il est impératif que l'Etat accorde à chacun un niveau de subsistance minimum. Lorsqu'il affirme qu'« un sentiment d'insécurité chronique s'oppose au bien-être social autant qu'à la productivité industrielle " ${ }^{16}$, Rowntree illustre bien la liaison que l'on établit désormais entre protection sociale et efficacité économique. La réflexion des enquêteurs sociaux ébranle les convictions victoriennes et justifie un réaménagement en profondeur des mécanismes d'assistance, 
inscrivant l'action de l'Etat dans une dynamique nouvelle. Elle suggère finalement une nouvelle conception de la régulation juridique et de l'action étatique : échapper à la logique dissuasive du droit punitif pour donner à la loi un contenu plus positif revient à admettre que la société tout entière doit agir sur elle-même. Ce nouveau paradigme va séduire tous ceux qui, dans le sillage du New Liberalism notamment, chercheront à tempérer les effets de la liberté contractuelle et du cash nexus; tous ceux qui, après Thomas Hill Green, adhéreront à une théorie organique de la société (Hobson, Hobhouse). L'idée fondamentale sera alors d'instrumentaliser le principe de responsabilité collective en instaurant des mécanismes protecteurs alternatifs à la Poor Law et étrangers à la logique du mérite. Au-delà des dispositifs d'assurance sociale (qui se mettent en place, pour le chômage et la maladie, dès 1911), l'assistance elle-même doit désormais apparaître comme une institution normale, débarrassée des procédés intimidants qui dissuadaient naguère d'y recourir. Là encore, les observateurs sociaux ont notablement contribué à restaurer la dignité du pauvre en plaidant pour une assistance universelle, inscrivant dans le registre du droit (social) ce qui relevait jadis de la sanction ou des devoirs (individuels). "C'est afin de préserver la dignité de tous que ceux qui n'ont pas besoin d'argent doivent aussi avoir le droit d'obtenir une pension [de retraite]» ${ }^{17}$ écrivait Booth en 1903 pour signifier son hostilité à un dispositif stigmatisant. Un système de ce type, à la fréquentation duquel n'est attaché aucun stigmate, sera mis en place dès 1908 (Old Age Pension Act) puis généralisé trois ans plus tard. Dans l'entre-deux-guerres encore, ce sont les enquêteurs sociaux, Herbert Tout, David Caradog Jones ou Arthur Bowley, qui chercheront à expurger de la politique sociale tout relent victorien. "L'idée selon laquelle les secours exercent une influence pernicieuse en cas de chômage est démentie par notre enquête. C'est le chômage lui-même qui exerce cette influence démoralisante - sentiment d'inutilité, oisiveté forcée, absence d'opportunité de travail - qui blesse la fierté du travailleur et lui fait peur " ${ }^{18}$. En inversant la causalité tenue pour évidente au cours du XIXème siècle, la réflexion sociale démontre là encore l'inanité de la philosophie sociale victorienne. Ce n'est pas l'assistance qui corrompt la «moralité » du travailleur désœuvré, mais c'est bien le chômage prolongé qui altère ses capacités. Au cours de la première moitié du XXème siècle, les observateurs sociaux ont ainsi fourni aux nouvelles élites politiques le cadre théorique qui allait justifier l'intervention croissante de l'Etat. La responsabilité de ce dernier sera clairement affirmée en matière de traitement de l'enfance (Children's Act de 1908); comme elle le sera à des degrés divers s'agissant du chômage, de la vieillesse et de la maladie. Parce qu'il appréhende la pauvreté à partir du critère de "l'efficience physique » et de l'apport nutritionnel minimum (suivant en cela les enseignements du diététicien américain Atwater), Rowntree sera l'un des principaux artisans de cette réorganisation du système d'assistance autour du paradigme du besoin. Il sera le propagandiste infatigable d'une politique sociale prenant en charge les besoins fondamentaux des individus et assurant à chacun un «niveau de subsistance » minimum. C'est pourquoi il défendra le principe d'un salaire minimum protégeant le travailleur contre les méfaits d'une concurrence exacerbée, et prendra parti en faveur du Coal Mines Act (1912), qui concrétisera cette revendication dans le secteur minier. De la même façon, il plaidera sans relâche pour que les prestations sociales répondent effectivement aux besoins des plus vulnérables : en 1918, dans The Human Needs of Labour, il montrera que le salaire minimum doit être complété par des allocations familiales à destination des familles nombreuses; lorsqu'il renouvellera son enquête de terrain à York, en 1936, il s'attachera à montrer 
que si le processus de paupérisation a changé, la pauvreté n'est pas vaincue. Il soulignera particulièrement l'impact du chômage sur les jeunes adultes et ses répercussions sur les jeunes enfants; il mettra en évidence l'insuffisance des prestations offertes aux personnes âgées ${ }^{19}$. Mais c'est sans doute en participant à la Commission Beveridge de 1941-42 que Rowntree (mais aussi Bowley) pourront exercer l'influence la plus décisive sur les orientations de la politique sociale et sur l'architecture d'un projet explicitement engagé sur «le chemin vers la libération du besoin ». A cet égard, le Rapport Beveridge, Social Insurance and Allied Services, couronne en quelque sorte l'évolution entamée en 1908 : la filiation avec le travail des enquêteurs sociaux est explicitement soulignée par Beveridge, de même que la nécessité de répondre aux « besoins » de l'existence humaine. «Au cours des années en question, des enquêteurs impartiaux ont procédé scientifiquement à l'étude sociale des conditions de vie dans certaines des principales villes de Grande-Bretagne, y compris Londres, Liverpool, Sheffield, Plymouth, Southampton, York et Bristol. Ils ont déterminé quelle était, dans chacune de ces villes, la proportion de la population dont le niveau de vie demeurait au-dessous du standard jugé indispensable à l'existence humaine, et ils ont recherché les causes et l'étendue de cette insuffisance. (...) L'abolition du besoin exige une double redistribution des revenus, par le moyen de l'assurance sociale et en tenant compte des exigences familiales $"{ }^{20}$. La suspension du gain (maladie, chômage), son insuffisance (charges familiales) ou son extinction (vieillesse) définissent des risques sociaux et autant de situations de besoin qui doivent désormais être traitées dans le cadre de l'assurance sociale et de l'assistance nationale. L'essentiel de la législation d'après-guerre visera donc à éliminer toutes les manifestations de l'insécurité sociale, et le système d'assistance de 1948 abrogera définitivement la loi des pauvres en reconnaissant à chacun le droit d'obtenir de la collectivité des moyens d'existence minima, indépendamment de toute appréciation des « mérites » individuels. Pour essentielle qu'elle ait été dans l'émergence du Welfare State britannique, la réflexion des pères fondateurs de la science sociale anglaise n'est pas exempte de toute ambiguïté. Cette ambiguïté reflète en réalité une tension qui traverse la sociologie britannique de la première moitié du siècle: tandis que les approches "quantitatives" soulignent la dimension structurelle de la pauvreté, les approches plus «qualitatives", tirant vers la sociologie (ou l'anthropologie) culturelle, font ressortir l'incompétence du pauvre, son éloignement coupable par rapport aux valeurs de la classe dominante. Sans lui accorder le même potentiel explicatif, les auteurs esquissent en fait les contours d'une "culture de la pauvreté", au sens que l'anthropologie américaine des années 1960 donnera à ce terme: une "vision du monde », un "mode de vie stable transmis d'une génération à l'autre " ${ }^{21}$. La réflexion se retourne alors vers le pauvre qui, enfermé dans un univers mental alternatif, est en proie au fatalisme. Il est significatif par exemple que, tout en insistant sur les effets dramatiques du sous-emploi, Booth achève sa volumineuse enquête sur la nécessité d'intensifier le «sentiment de responsabilité individuelle» ${ }^{22}$. Il lui semble effectivement nécessaire de briser le "piège à pauvreté » qui maintient quantité d'individus en situation de passivité. L'idée selon laquelle la pauvreté est reproduite par la culture qui lui a donné naissance est avancée plus nettement encore par Lady Bell ou Margaret Loane ; celle-ci décrit avec ironie les conséquences funestes du comportement des pauvres : «Peu de personnes réalisent l'importance du prélèvement que les pauvres opèrent sur leurs propres ressources; les sommes gaspillées par insouciance, ignorance, crédulité ou par une suspicion hors de propos, et celles qui le sont par un 
manque de tempérance, de clairvoyance ou d'autodiscipline (...) pourraient fournir un excèdent budgétaire temporaire même à un gouvernement progressiste " ${ }^{23}$ Le pauvre fait ici l'objet d'une représentation discriminatoire: l'altérité et la différence sont interprétées comme des insuffisances ou des manques, un manque de compétence ou de contention. La proximité avec la philosophie sociale de 1834 est assez troublante, d'autant plus qu'on la retrouve dans l'œuvre de Rowntree, pourtant ouvertement favorable à l'extension de la protection sociale. Tous les travaux de Rowntree sont paradoxalement marqués par une tension entre deux formes de pauvreté, la première échappant à l'emprise du pauvre, tandis qu'il emporte seul la responsabilité de la seconde. Au sein de la population dont les besoins minima ne sont pas assurés, l'enquêteur distingue en effet la "pauvreté primaire ", c'est-à-dire les personnes dont les ressources sont à l'évidence insuffisantes, et la " pauvreté secondaire » résultant des "causes immédiates que sont la boisson, les paris et le jeu; une gestion familiale négligente ou ignorante et d'autres dépenses inconsidérées " ${ }^{24}$. Le biographe de Rowntree expliquera que celui-ci réserve peu de développements à ce type de pauvreté (5 pages dans l'enquête de 1901), de peur de glisser dans le commentaire moralisateur ${ }^{25}$. Mais il s'agit toutefois d'un thème récurrent dans l'ensemble de son œuvre : en 1951 encore, dans son ultime ouvrage, il regardera les loisirs populaires avec l'œil du moraliste. Les paris, la fréquentation des champs de courses ou des pub, le cinéma ou le dancing, toutes ces activités qui ne favorisent pas le «développement mental» constituent "un nouveau problème social qui appelle une solution urgente " ${ }^{26}$. La "sociologie» de ces auteurs s'avère donc quelque peu ambiguë car le pauvre est toujours, peu ou prou, complice de sa déchéance. Leur réflexion oscille en réalité entre deux registres analytiques, celui de la responsabilité individuelle, duquel ils ne parviennent pas à se dégager complètement, et celui des causes objectives. Cette ambivalence est tout à fait symptomatique de la résistance du schéma interprétatif issu du XIXème siècle. Derrière la neutralité d'un vocabulaire qui se veut scientifique - les « classes » A, B, C, etc. de Booth ; la " pauvreté secondaire » de Rowntree - réapparaît le principe victorien de division du monde social : on prête au pauvre des aptitudes et des motivations différentes de celles des autres citoyens. Ainsi, les auteurs qui ont le plus contribué à promouvoir l'idée d'un «droit à l'existence" restent finalement prisonniers d'un cadre analytique qui conçoit le statut d'assisté avec une relative méfiance. "L'octroi de l'assistance sera également subordonné, en ce qui concerne la conduite des intéressés, à toutes conditions qui paraitront de nature à hâter le rétablissement de la capacité de gain " ${ }^{27}$ écrira par exemple Beveridge dans son rapport de 1942. Le retour du refoulé L'historiographie, qui présente volontiers l'évolution de la politique sociale anglaise comme une marche en avant couronnée par le "consensus keynéso-beveridgien", offre une représentation probablement simplifiée de la construction de la citoyenneté sociale. Cette représentation suggère que la "révolution» thatchérienne est venue remettre en cause les responsabilités sociales incombant à l'Etat depuis le second après-guerre. Ce faisant, elle surestime la stabilité du système façonné par le gouvernement Attlee (1946-51), et mésestime la résistance sur le long terme d'une attitude critique et suspicieuse à l'égard des assistés sociaux. Il n'est pas excessif de prétendre qu'en matière de traitement de la pauvreté l'apparition du Welfare State constitue une anomalie, qui tranche avec les mesures prises avant guerre autant qu'avec les débats suscités par son développement ultérieur. Même si elles ne correspondaient pas exactement aux recommandations de Beveridge, les dispositions prises dans le domaine de l'assistance publique en 1948 s'opposaient 
résolument aux procédures vexatoires de la loi des pauvres - encore que les mécanismes mis en place visaient explicitement à inciter les bénéficiaires à reconquérir leur indépendance (les prestations sont nettement moins généreuses que les allocations d'assurance offertes aux travailleurs-cotisants, et placées sous conditions de ressources). Avant les années Thatcher, le système de protection sociale sera la cible de critiques convergentes qui rappellent étrangement les débats précédents la New Poor Law de 1834. En 1952 déjà, au lendemain des grandes mesures prises par le gouvernement Attlee, le Times se faisait l'écho des préoccupations des Conservateurs et annonçait la "crise de l'Etat providence ", suggérant aussitôt un repli de l'Etat sur des interventions prioritaires ${ }^{28}$. Le retour des Conservateurs au pouvoir (1951-64) se traduira effectivement par un ciblage de la politique sociale dérogeant à l'universalisme beveridgien. D'autres critiques auront pour fondement l'éthique du travail, et pour point de mire la "démoralisation» du pauvre, sa passivité; elles se développèrent principalement autour de la thématique du "piège à chômage ", promise à une glorieuse postérité au cours des décennies suivantes. Dès les années 1960 en effet, certaines études montreront que les prestations complémentaires accordées par l'institution d'assistance (devenue la Supplementary Benefits Commission) aux chômeurs disposant de ressources insuffisantes les plaçaient dans une situation plus favorable que celle d'un travailleur ordinaire. Cette démonstration viendra étoffer l'argumentaire de ceux qui, dans l'entourage du parti conservateur notamment, allaient instruire le procès du pauvre et "blâmer la victime ». En développant son analyse du "cycle de pauvreté », Keith Joseph, l'un des inspirateurs de Thatcher, proposera par exemple une nouvelle formulation de la théorie de la «culture de la pauvreté »: une éducation parentale défaillante, un faible capital culturel, alliés à de mauvaises conditions de logement, favoriseraient l'apparition d'une pauvreté héréditaire. L'auteur en appelait alors aux valeurs victoriennes de la famille et suggérait une réhabilitation de la politique familiale. Le thème de la «culture de la dépendance " permettra plus tard à Margaret Thatcher d'articuler cette première approche avec le schéma du pauvre «non méritant ». L'orientation qui sera donnée à la politique britannique au cours des années 1980 est souvent présentée comme une "révolution" introduisant une césure nette avec les occurrences passées, et notamment le fameux «consensus » d'après-guerre fondé sur la protection sociale et une politique de plein emploi. Certes, en adoptant les préceptes du monétarisme ou de l'économie de l'offre, Geoffrey Howe ou Keith Joseph s'opposaient clairement au travaillisme ou au torysme des années 1950-60 - converti à l'interventionnisme pour s'attacher le soutien des masses - et souhaitaient propager un nouveau «climat économique » où chacun serait soumis à l'arbitrage incessant du marché - une " société sans classe " où les statuts ne seraient jamais figés ${ }^{29}$. Mais si cette nouvelle source d'inspiration a eu une traduction immédiate en matière de politique économique (la lutte contre l'inflation), de réforme fiscale, ou dans la gestion du dossier des syndicats, cela est moins net s'agissant du traitement de la pauvreté. Les mesures sévères qui furent prises dans ce domaine ne s'inspirent pas directement des dogmes du libéralisme "façon Thatcher ", ni du Workfare américain, mais de la représentation dévalorisante du pauvre héritée du XIXème siècle. Leur manque d'originalité sera d'ailleurs garant de leur efficacité politique : elles tireront leur force de la prégnance de réactions sociales de défiance ou d'hostilité à l'égard des assistés sociaux. Les Conservateurs vont en effet entreprendre un travail de reconstruction des catégories de la pauvreté légitime: dans l'ensemble de la population tributaire des secours de la 
collectivité, les jeunes et les chômeurs valides, dont le comportement déroge à l'ethos néo-victorien que le gouvernement veut imposer, ont été requalifiés comme " mauvais pauvres" et exclus des dispositifs de droit commun. Dès 1980, les allocations d'assurance chômage ont été réduites, le gouvernement donnant en outre mandat à des enquêteurs (investigators) de dénicher les fraudeurs, comme il le fit naguère sous l'égide de l'Anomalies Act de 1934. Pour inciter les travailleurs désœuvrés à retrouver un emploi, les chômeurs perdront le bénéfice des prestations complémentaires accordées en fonction du nombre d'enfants à charge ; et en 1986, le Social Security Act diminuera encore le montant des allocations d'assistance (income support) en direction des chômeurs et des jeunes - dont la prise en charge incombera désormais aux familles. Depuis la fin des années 1980, ces deux catégories d'assistés sont soumises à une surveillance très étroite, subordonnant le versement des allocations à la recherche effective d'un emploi ou à la poursuite d'une action de formation. Comme en 1920 lorsque les responsables des bourses du travail eurent pour mission de prévenir les abus en s'assurant de la réalité de la situation de chômage, les allocataires doivent prouver qu'ils sont effectivement «disponibles pour un travail» ${ }^{30}$. Point d'orgue de cette politique: en 1996, le Jobseeker's Act supprime le principe de l'allocation de chômage comme un droit. Désormais, assurance-chômage et aide sociale à destination des chômeurs sont remplacées par une prestation discrétionnaire unique (jobseeker's allowance), placée sous conditions de ressources et destinée aux seules personnes qui souscrivent l'engagement formel de mener une action déterminée et planifiée pour retrouver un emploi. Les prestations de chômage sont ainsi (re)devenues la contrepartie d'un contrôle des comportements. Au pauvre "non méritant» on applique donc des techniques assistancielles dissuasives destinées à tester la réalité de ses besoins; à la baisse des prestations, qui incite à "s'en sortir" (au besoin dans l'économie parallèle), s'ajoutent les procédés discriminatoires et humiliants auxquels recourent les bureaux d'assistance. "L'objectif premier de notre loi des pauvres actuelle est de permettre aux gens de survivre tout en vérifiant que leurs demandes sont fondées » expliquera Paul Harrison au terme d'une enquête menée dans le quartier londonien de Hackney, avant d'ajouter que «cela implique une humiliation systématique des clients et conduit à priver certaines personnes de leurs droits ${ }^{31}$. Aux régulations étatiques on substitue, par ailleurs, des solutions passant par le marché et l'initiative privée. Favoriser l'intégration économique du plus grand nombre, faire appel à leur « sens caché » des affaires, comme dit David Robins ${ }^{32}$, et créer un climat de croissance auto-entretenue à partir du secteur privé: tels sont désormais les instruments majeurs de la lutte contre la pauvreté. Deakin et Edwards soulignent par exemple la confiance excessive accordée aux entreprises pour résoudre les problèmes sociaux des inner cities anglaises. Dans ce cadre, l'appel à l'initiative privée n'apparaît pas seulement comme une solution technique permettant d'alléger les charges de l'Etat en transférant certaines responsabilités aux entreprises: la culture d'entreprise est pensée comme un contre-modèle à la "culture du pauvre", capable d'en réduire l'emprise en favorisant l'adhésion des pauvres aux valeurs de la classe moyenne. « On ne peut s'empêcher de conclure ", affirment ces auteurs, " que, pour le gouvernement, le problème des centres-villes se réduit dans une certaine mesure au problème des personnes jugées inadaptées - l'antithèse en fait de l'entrepreneur, dynamique, efficace, autonome, combatif ... et conservateur " ${ }^{33}$. "Le véritable objectif du Welfare State (...) c'est d'apprendre aux gens à s'en passer " ${ }^{34}$ : cette formule lapidaire préfigurait les axes de la politique sociale thatchérienne; elle conviendrait aussi 
parfaitement pour définir l'objectif assigné à l'assistance tout au long du XIXème siècle, et encore dans les années 1920-30. Dans les groupes de réflexion voués à la diffusion de la doxa libérale, en particulier l'Institute of Economic Affairs (fondé en 1957), le système de protection sociale et l'attitude des assistés sociaux faisaient l'objet de critiques récurrentes qui gagnaient une large audience, en raison notamment de l'écho qu'elles recevaient dans la presse. Et au cours des années 1970, plus d'un quart de la population, soit deux ou trois fois plus que dans les autres pays européens, estimait que la pauvreté n'existait pas ou que les pauvres étaient directement responsables de leur disgrâce ${ }^{35}$. Une perception de la réalité sociale aussi communément partagée explique sans doute le succès de la rhétorique conservatrice. Lutter contre les abus individuels au nom du travailleur « respectable ", floué dans ses droits de contribuable, encourager les "paresseux » à travailler afin de défendre «l'héritage » et la dignité du citoyen britannique : ces mots d'ordre allaient finalement permettre de dégager un consensus contre les droits sociaux. Cet article suggère toutefois que l'adhésion des Britanniques aux campagnes lancées dans les années 1980 contre les "parasites" et les « combinards» traduit probablement moins l'influence immédiate de la vulgate néolibérale que la présence d'un trait culturel persistant laissant à l'individu la responsabilité de son sort. A cet égard, la « révolution thatchérienne » ressemble fort à une tentative pour saisir le présent dans une grille analytique (dé)passée, pour réactualiser et instrumentaliser cette perception dévalorisante de la pauvreté assistée qui traverse l'histoire sociale britannique depuis le XIXème siècle au moins ; bref, pour ériger le sens commun en principe d'action politique. Dès lors, la véritable nouveauté de cette période en matière de traitement de la pauvreté réside sans doute dans la réduction des prestations allouées à des catégories de bénéficiaires "méritants ", en particulier les retraités les plus modestes; elle traduit une dévaluation du critère du besoin que les sciences sociales étaient parvenues à hisser jusqu'au cœur du Welfare State d'après-guerre.

\section{NOTES}

1. Cf. N. Bosanquet, « Poverty under Thatcher », in M. Loney, D. Boswell, J. Clarke (ed.), Social Policy and Social Welfare, Buckingham, Open University Press, 1996, pp. 183-196. 2. Cf. T.H. Marshall, « The Welfare State : A Sociological Interpretation », Archives Européennes de Sociologie, ii, 1961, pp. 284-300.

3. F.M. Eden, The State of the Poor (1797), Londres, Frank Cass, 1966, vol. 1, p. 467.

4. E. Chadwick, « The New Poor Law », Edinburgh Review, vol. 63, 1836, pp. 500-501.

5. J. Bentham, Le panoptique, Paris, ed. Belfond, coll. «L'échappée », 1977, pp. 38-39.

6. Cf. F-X. Merrien (dir.), Face à la pauvreté, Paris, Les éditions de l'atelier/Editions ouvrières, coll. « Le social en acte », 1994, pp. 99-135. L'auteur utilise l'expression « pauvreté volontaire », mais celle-ci ne paraît pas pleinement satisfaisante. En effet, dans le schéma intellectuel des réformateurs victoriens, la « pauvreté » n'existe pas : elle est appelée à se dissoudre dans l'indépendance laborieuse qui en est l'antithèse. 
Seule subsiste l'indigence, c'est-à-dire le statut limite de ceux qui n'ont d'autre échappatoire que la workhouse.

7. Poor Law Commission, circulaire de 1835 , citée par J.H. Treble, Urban Poverty in Britain 1830-1914, Londres, Routledge, 1990, p. 140.

8. Lyndoch Gardiner, cité par H. Bosanquet, Social Work in London, 1869-1912 (1914), Brighton, The Harvester Press, 1973, p. 41.

9. Cf. B. et S. Webb, English Local Government, vol. 10 « English Poor Law Policy » (1929), Londres, Frank Cass, 1963, pp. 304-307.

10. Cf. H. Mayhew, London Labour and the London Poor (1861), 4 vol., Londres, Frank Cass, 1967 ; G. Sims, How the Poor Live, Londres, 1883 ; A. Mearns, The Bitter Cry of Outcast London (1883), Leicester, Leicester University Press, coll. « Victorian Library », 1970.

11. C. Booth, Life and Labour of the People of London (1902-03), 17 vol., New York, Augustus M. Kelley Publishers, 1969, « Poverty Series », vol. 1, p. 147.

12. Cf. B.S. Rowntree, Poverty : A Study of Town Life, Londres, Macmillan \& Co, 1901, pp. 136-137 ; sur le même thème voir aussi C. Booth, op. cit., "Industry Series », vol. 5, pp. 319-339.

13. Cf. B.S. Rowntree, Poverty : A Study of Town Life, Londres, Macmillan \& Co, 1901, pp. 136-137 ; sur le même thème voir aussi C. Booth, op. cit., « Industry Series ", vol. 5, pp. 319-339.

14. B.S. Rowntree, op. cit., pp. 133-34.

15. Florence (Lady) Bell, At the Works (1907), Londres, Virago Press, 1985, p. 86. 16. B.S. Rowntree, «Charte du Welfare » édictée en 1917 au sein du Ministère des Munitions, cité par A. Briggs, A Study of the Work of Seebhom Rowntree, Londres, Longmans, 1961, p. 130.

17. C. Booth, op. cit., « Final Volume », p. 144.

18. A. Bowley, H. Clay, B.S. Rowntree et alii, The Third Winter of Unemployment, Londres, Macmillan, 1923, p. 11.

19. Cf. B.S. Rowntree, Poverty and Progress. A Second Social Survey of York, Londres, Longmans, Green \& Co, 1941, en particulier le chap. 4 « Primary Poverty », pp. 101-120. 20. W. Beveridge, Social Insurance and Allied Services, Londres, 1942, §11; reproduit dans E. Milhaud, Le plan Beveridge, Genève, Les annales de l'économie collective, 1943, p. 18. C'est nous qui soulignons.

21. O. Lewis, Les enfants de Sanchez (1961), trad. C. Zins, Paris, Gallimard, 1963, pp. 25 et 29 .

22. C. Booth, op. cit., « Final Volume », p. 21

23. M.E. Loane, Neighbours and Friends, Londres, 1910, p. 278.

24. B.S. Rowntree, Poverty. A Study of Town Life, op. cit., p. 142.

25. A. Briggs, A Study of the Work of Seebhom Rowntree, op. cit., p. 33.

26. Cf. B.S. Rowntree et G.R. Lavers, English Life and Leisure, A Social Study, Londres, Longmans, Green \& Co, 1951.

27. W. Beveridge, Social Insurance and Allied Services, op. cit. (\$369), p. 46.

28. Cf. The Times, 25 février 1952, p. 7.

29. Cf. F. Poirier, Génération Thatcher. La culture politique de l'Angleterre, Vincennes, Presses Universitaires de Vincennes, 1992.

30. Cf. N. Whiteside, Bad Times, Unemployment in British Social and Political History, Londres, Faber \& Faber, 1991.

31. P. Harrison, Inside the Inner City (1983), Londres, Penguin Books, 1992, p. 142. 
32. D. Robins, Tarnished Vision, Oxford, Oxford University Press, 1992, p.125.

33. N. Deakin, J. Edwards The Entreprise Culture and the Inner City, Londres, Routledge, 1993, pp. 48-49.

34. A. Peacock, The Welfare Society, Londres, Institute of Economic Affairs, 1961, p. 27.

35. Cf. M. Loney, D. Boswell, J. Clarke (ed.), Social Policy and Social Welfare, op. cit., pp. 58-82.

INDEX

Index géographique : Royaume Uni

Index chronologique : $19 \mathrm{e}$ siècle

Mots-clés : pauvreté, Etat providence, économie politique, généalogie/ socio-génèse, histoire 